\title{
Los Padres de Familia y el Logro Académico de los Adolescentes de una Secundaria en Milpa Alta, Ciudad de México
}

\author{
Claudia A. Hernández ${ }^{(1)}$, Carlos A. Cárdenas ${ }^{(1)}$, Paola O. Romero ${ }^{(2)}$ y Marycarmen Hernández ${ }^{(3)}$ \\ (1) Instituto Politécnico Nacional, Unidad Profesional Interdisciplinaria de Ingeniería y Ciencias Sociales y \\ Administrativas (UPIICSA), Sección de Estudios de Posgrado e Investigación. Av. Té 950, Iztacalco, \\ Granjas México, 08400, México D.F. - México (e-mail: al9505@gmail.com, \\ carlos.cardenas.do@gmail.com). \\ (2) Centro de Estudios Superiores en Educación (CESE), Benito Juárez 108, colonia Albert, delegación \\ Benito Juárez, 03560, Ciudad de México, D.F. - México (e-mail: pao.rom.t@gmail.com). \\ (3) Instituto Tecnológico de Gustavo A. Madero, Calle 608 No. 300 y Av. 412, Col. San Juan de Aragón, \\ Delegación. Gustavo A. Madero, C.P. 07470, México, D.F. - México (e-mail: acad_gamadero@tecnm.mx).
}

Recibido Sep. 13, 2016; Aceptado Nov. 9, 2016; Versión final Feb. 15, 2017, Publicado Jun. 2017

\begin{abstract}
Resumen
El objetivo del presente artículo es mostrar los resultados obtenidos de la percepción que tienen los estudiantes de una secundaria ubicada en la delegación Milpa Alta en la Ciudad de México respecto al trabajo cotidiano del acompañamiento académico que realizan sus padres orientado al fortalecimiento del éxito académico. Es un estudio cuantitativo y transversal, la obtención de datos se realizó a través de un cuestionario, la muestra fue de 450 jóvenes, y se realizó un análisis factorial que obtuvo tres principales factores que representan el logro: a) supervisión académica, b) autoestima y motivación y c) castigos y atención a citatorios importantes. Se concluye que la supervisión académica de los padres varones es mayor que el de las madres. Además las mamás son las que más influyen en la motivación y autoestima de sus hijos. Por último las mujeres adolescentes son las que reciben en mayor medida los castigos comparado con los varones.
\end{abstract}

Palabras clave: adolescentes, padres de familia, logro académico, profesores, éxito académico

\section{The Parents and the Academic Achievement of Teenager Students in a High School of Milpa Alta, Mexico City}

\begin{abstract}
The aim of the present article is to show the results obtained from the perception of the students of a secondary school located in Milpa Alta delegation in Mexico City, regarding the daily work of the academic monitoring carried out by their parents, aimed at strengthening academic success. The study was a quantitative and cross-sectional study, the data was obtained through a questionnaire, the sample consisted of 450 youngsters, and an exploratory factor analysis was carried out to obtain three factors that represent the academic achievement: a) academic supervision, b) self-esteem and motivation and c) punishment and attending meeting requests from the school. It is concluded that the academic supervision of the parents is higher than that of the mothers. Also, the mothers are those that have more influence in the motivation and self-esteem of their children. Finally the younger women are the ones that receive more punishments compared to that of the young men.
\end{abstract}

Keywords: teenagers, parents, academic achievement, teachers, academic success 


\section{INTRODUCCIÓN}

El Plan Nacional de Desarrollo 2012-2018 reconoce que la educación de los adolescentes es responsabilidad compartida entre los diferentes actores que participan en el contexto escolar, por lo que las reformas educativas pretenden implementar acciones para que los directivos, profesores y padres de familia se involucren y generen acciones que permitan tomar decisiones que coadyuven a perseguir el incremento del nivel educativo así como el éxito académico de los alumnos.

Se dice que el logro académico no dependen únicamente del sistema escolar sino de la interacción de diversos factores que se relacionan en ocasiones entre sí (García, et. al., 2013; López, et. al, 2011) . El abordar el tema del logro académico permite analizar diferentes problemas a los que se enfrentan los jóvenes en la escuela, además reflexiona sobre las variables que influyen en el fenómeno como es la motivación hacia la escuela (Areepattamannil, 2014; Carvallo, et. al., 2007; Gutman y McLoyd; 2000; Kim, et. al., 2015; Loose, 2008). Una forma que se utiliza comúnmente para medir el logro académico de las personas, son los grados académicos, sin embargo, proporcionan poca información sobre los procesos que fomentan el éxito académico (Stanard, et. al, 2010).

Dentro del estudio sobre el logro académico se encuentra la familia que debe responsabilizarse en guiar al estudiante en la búsqueda de adquirir autoestima y aprender a sociabilizar con las personas. Se tienen investigaciones que relacionan las variables sociodemográficas del entorno familiar con elementos que determinan el éxito en la escuela (Chaparro, et. al., 2016; Crede, et. al., 2015; García, et. al., 2013). Además, se dice que debe existir una participación solidaria entre las familias y los centros educativos que contribuyan al desarrollo integral de los niños (Coronado, et. al, 2011).

Se tienen hallazgos expuestos por Kristjánsson y Sigfúsdóttir (2009) que indican que si existe una relación entre el apoyo de los padres y las variables como la supervisión, el tiempo que se pasa con ellos y el logro académico. Por su parte, Latunde (2017) y Stanard et al. (2010), muestran que la familia juega un rol importante en el desarrollo del adolescente ya que permite tener fuertes lazos familiares, la ausencia de éstos aumenta la posibilidad de que se reciban malas influencias. En este mismo sentido, Henry et al. (2011), halló que la participación en específico del papá tiene efectos positivos en los resultados académicos de los adolescentes. Ahora bien, Cho y Campbell (2010) señalaron que el entorno familiar influyen en el comportamiento de un niño en la escuela, esto es porque la presencia de los padres propicia a que ellos visualicen la meta, se sabe que los padres que están presentes durante la vida escolar de los hijos contribuyen de forma adecuada para que los jóvenes visualicen sus metas académicas, pero al contrario cuando hay ausencia los efectos no son favorables, se presentan signos de mal comportamiento y bajo rendimiento académico. Sin embargo, se sabe que los padres pueden pensar que sus hijos pueden sacar adelante su vida escolar, esto provoca que los adolescentes sean abandonados y por ende la responsabilidad en varias ocasiones pasa a los profesores quienes necesitan poner atención a muchos jóvenes que requieren de su apoyo (Simon, 2004). Por último, en México se han realizado estudios con adolescentes que han indagado sobre la percepción que tienen los jóvenes sobre su autoconcepto académico para identificar sus percepciones sobre el concepto de sí mismos como estudiantes, cualidades sobre el desempeño académico y competencias académicas, no se contempló en la investigación la variable de los padres (Ibarra, et. al., 2016).

Por lo anterior, el objetivo del artículo es analizar la percepción de los estudiantes de una secundaria ubicada en la Delegación Milpa Alta en la Ciudad de México con relación al acompañamiento académico que llevan a cabo sus padres orientado al éxito académico.

\section{ANTECEDENTES DE LA LITERATURA}

Los padres y la familia tienen la encomienda de monitorear el comportamiento de los jóvenes en el hogar y en la escuela, además de conocer sobre las metas, motivaciones académicas y las relaciones interpersonales que los adolescentes tienen en la escuela (Henry, et. al., 2011; Kilian, et. al., 2013). De acuerdo con Kim, et. al. (2015), indica que cuando los papás muestran interés con relación al logro académico de los hijos, la motivación de los niños aumenta. Además, las formas de acompañamiento familiar según García, et. al., (2013), tienen relación con el desarrollo de habilidades y aptitudes, el manejo de las emociones y apoyo en las tareas escolares. Se dice que la familia trabaja como factor de protección para la salud física, emocional y regula ciertos comportamientos psicosociales que son detonantes del éxito en la escuela, todo lo anterior debe tomarse en cuenta ya que en la adolescencia es una etapa en donde influyen los componentes emocionales y motivacionales (Braun, et. al., 2017). Por su parte, Ricard y Pelletier (2016), indican que al parecer la motivación para aprender proviene de las relaciones reciprocas en la escuela y que los padres en ocasiones no tiene tanta presencia, como la pueden llegar a tener los amigos de la escuela. 
Se ha señalado que el nivel académico de los jefes de familia influye en el nivel de involucramiento en la vida académica de los adolescentes, es decir a mayor nivel de escolaridad toman mayor participación crítica que aquellos que demuestran menos niveles educativos (Räty, 2010). Ahora bien, según Toro, et.al. (2013) los padres de familia tienden a participar en la formación académica de los hijos porque tiene la ilusión de que accedan a los estudios universitarios y de esta forma puedan encontrar un mejor trabajo. Se tienen hallazgos que indican que los padres que son democráticos y permisivos coadyuvan a que sus hijos utilicen en mayor medida estrategias de aprendizaje autorregulado, en cambio se dice que los hijos de padres que son clasificados como autoritarios y negligentes no permiten que se desarrolle el aprendizaje autónomo (Cerezo, et. al, 2015). Autores como Lazaridou y Gravani (2015) mencionan que la participación de los padres incrementa las posibilidades de éxito académico, además apoya en solventar los obstáculos de la adolescencia. Se tiene evidencia de que las expectativas de los padres son un elemento que influye en la toma de decisiones en las elecciones académicas a futuro de los jóvenes (Rao y Mehta, 2017).

La participación de la familia en el éxito académico de los jóvenes es trascendental en el éxito y logro académico de los adolescentes (Arostegui, Beloki y Darretxe, 2013). Por otra parte, se pone énfasis en la importancia que tiene el desarrollo de la autoestima de parte de los padres que contribuye al rendimiento escolar (Booth y Gerard, 2011). Al parecer el logro académico de los jóvenes está relacionado con las expectativas académicas de ellos (Wilder, 2014).

\section{METODOLOGÍA}

La escuela secundaria se encuentra ubicada en la Ciudad de México en la Delegación Milpa Alta, actualmente cuenta con una población aproximada de 480 jóvenes. Se estructuró un cuestionario para recabar datos integrado por 17 preguntas, los ítems fueron elaborados considerando la toma de opinión de tres profesoras que imparten clase en los tres grados y que identifican el rol de los tutores que en diversas ocasiones son los padres de familia, además los enunciados responden a las variables que se tienen en el contexto escolar, pero algunas otras de las variables se detectaron en el análisis de la literatura, se empleó la escala tipo Likert, en dónde $1=$ totalmente en desacuerdo y $5=$ totalmente de acuerdo. La escuela cuenta aproximadamente 480 alumnos, se calculó la muestra considerando un nivel de confiabilidad del $95 \%$, con un $p=50 \%$ y $q=50 \%$, dónde $\mathrm{p}$ señala la probabilidad de que los encuestados señalen estar de acuerdo con los enunciados y $q$ la probabilidad de estar en desacuerdo, obteniendo una $n=214$ con un margen de error de 5.30\%, pero la muestra productora de datos fue de 450 estudiantes, lo que significó casi totalidad la población. Cabe aclarar que la validación del instrumento se llevó a cabo mediante la aplicación de una prueba piloto del cuestionario a 25 adolescentes, se ejecutó la prueba de confiabilidad de escala con un coeficiente de Alfa de Cronbach de 0.855 que indica una alta consistencia entre los enunciados, por lo que está correlacionados entre sí. Es un método cuantitativo y transversal, el levantamiento de datos se realizó en febrero de 2016 . Se llevó a cabo un análisis factorial exploratorio, el cual arrojó una prueba del Kaiser Meyer Olkin $0.897>0.5$ que significa que los ítems tienen consistencia interna por medio de todos los elementos y por lo tanto están correlacionados entre sí. El método de la extracción fue por componentes principales y la reducción fue varimax, prueba de máxima verosimilitud. Se obtuvo como resultado tres factores, siendo el primero el que explica el $32.29 \%$ de la varianza total. La tabla 1 muestra los resultados del análisis factorial y los coeficientes de correlación.

\section{RESULTADOS Y DISCUSIÓN}

Se aplicaron 450 cuestionarios a estudiantes de una secundaria en la delegación Milpa Alta, de los cuales el $51 \%$ mujeres y el $49 \%$ hombres, de los cuales el $36 \%$ dijo estar cursando el primer semestre, el $39 \%$ segundo y el $25 \%$ el tercer año de secundaria, además sus edades oscilan entre los doce y quince años. El $81 \%$ de los adolescentes señalaron que su tutor es su mamá, el $14 \%$ su papá, el $2 \%$ los abuelos, el $1 \%$ los tíos y el $2 \%$ los hermanos.

\section{Factor 1: Supervisión Académica de los Tutores}

El $46 \%$ de los adolescentes afirmaron que sus tutores y sus padres revisan lo que llevan en la mochila, el $36 \%$ de los participantes indicaron que no, mientras que el $17 \%$ se mostró indiferente a la respuesta. Por otro lado, el $40 \%$ de los alumnos dicen que sus padres o tutores revisan de forma constante sus cuadernos y libros para conocer su avance académico, pero el 34\% dijo no estar de acuerdo. Además, el 33\% enfatizó que sus tutores asisten a la escuela de forma constante para preguntar sobre su situación escolar, el $40 \%$ indicó no estar de acuerdo y el resto no mostró tendencia. También el $50 \%$ dijo que sus tutores de forma frecuente los supervisan mientras realizan las tareas escolares, sin embargo, el $29 \%$ dijo que no, mientras que el $21 \%$ fue neutral en su respuesta. De igual forma, se les preguntó a los adolescentes si sus tutores les ayudan a realizar sus tareas escolares, el $48 \%$ de ellos indicaron que sí, pero el $30 \%$ señaló que no. 
Tabla 1: Análisis factorial exploratorio

\begin{tabular}{|c|c|}
\hline Variable & $\begin{array}{c}\text { Coeficiente de } \\
\text { correlación }\end{array}$ \\
\hline \multicolumn{2}{|l|}{ Factor 1. Supervisión académica de los tutores } \\
\hline 5. Revisión de la mochila del estudiante & 0.735 \\
\hline 4. Revisión de forma constante de los cuadernos y libros para identificar el avance del estudiante. & 0.719 \\
\hline 7. El tutor acude frecuentemente a la escuela para conocer la situación del estudiante. & 0.666 \\
\hline 3. Supervisión del tutor en la realización de tareas escolares. & 0.665 \\
\hline 16. Ayuda de parte del tutor en la realización de las tareas. & 0.594 \\
\hline 2. El tutor pregunta sobre cómo le fue al joven en la escuela. & 0.536 \\
\hline 9. El tutor conocer el horario del alumno. & 0.509 \\
\hline 10. El tutor inculca buenos hábitos de estudio. & 0.350 \\
\hline \multicolumn{2}{|l|}{ Factor 2. Autoestima y motivación } \\
\hline 12. El tutor ofrece de repetidas ocasiones muestras de cariño. & 0.759 \\
\hline 13. Motivación del tutor. & 0.749 \\
\hline 17. El tutor es un apoyo para el incremento de la autoestima. & 0.669 \\
\hline 11. El tutor estimula para que el estudiante sea puntual en la escuela. & 0.574 \\
\hline 15. El tutor muestra interés o en su caso preocupación por el avance en la escuela. & 0.565 \\
\hline 8. El tutor se preocupa que no falte nada de material para las actividades de la escuela. & 0.433 \\
\hline 1. La presencia de alguien en casa. & 0.420 \\
\hline \multicolumn{2}{|l|}{ Factor 3. Castigos y atención a los citatorios importantes } \\
\hline 12. Castigos de parte del tutor en caso de obtener malas calificaciones. & 0.716 \\
\hline 6. Los tutores siempre asisten a las juntas y atienden los citatorios. & 0.633 \\
\hline
\end{tabular}

En el estudio se indagó si los tutores preguntaban a los adolescentes si habían aprendido en la escuela y si les había ido bien, a lo que el $65 \%$ señaló que si y únicamente el $17 \%$ dijo que no y el $18 \%$ se mostró ni de acuerdo ni en desacuerdo. Asimismo, se indagó si los tutores conocían el horario escolar de los estudiantes, el $34 \%$ de los participantes dijeron estar en desacuerdo, mientras que el $47 \%$ dijo estar de acuerdo. Por último, se encontró que el $6 \%$ de los adolescentes señaló estar en desacuerdo que su tutor le inculca buenos hábitos de estudio mientras que el $84 \%$ cree que sus tutores se esfuerzan para que ellos obtengan hábitos relacionados con su forma de aprender, el $10 \%$ de los participantes se mostraron neutrales. Se realizó un modelo lineal generalizado del factor uno con respecto a las variables de género, año escolar y el tutor. El modelo arrojó en la prueba de contraste de Omnibus un $\mathrm{p}=0.002<0.05$ que indica que existe efecto significativo por lo menos en una de las variables del modelo (ver tabla 2), en el análisis de los efectos (ver tabla 3) se halló que el género obtuvo una $p=0.282>0.05$ que indica sin efecto significativo, el año escolar con una $p=0.066>0.05$ también sin efecto y el tutor con $p=0.005>=0.05$, aquí se encontró efecto significativo en la variable en donde se detectó que el mayor nivel de supervisión académica proviene del papá $(\bar{x}=27.76$, en segundo lugar la madre $(\bar{x}=26.89)$, en tercer lugar los hermanos $(\bar{x}=23.98)$, en cuarto lugar los abuelos $\overline{(x}=23.75)$.

Por otra parte, se elaboró una escala con los ocho ítems que comprenden el factor con un mínimo de 5 y un máximo de 40, con cuatro escalas que son de "muy bajo" al "alto", después se llevó a cabo una tabla de contingencia que permitió obtener el comparativo. Se halló que $87 \%$ de los jóvenes del primer año perciben como "buena" y "alta" la supervisión de sus tutores, por otro lado, el $76 \%$ de los adolescentes del segundo año siente que la atención de sus tutores se encuentra entre "buena" y "alta", por último el $81 \%$ de los alumnos del tercer semestre de igual forma señaló sentir la atención de sus tutores hacia su vida académica como "buena" y "alta". 


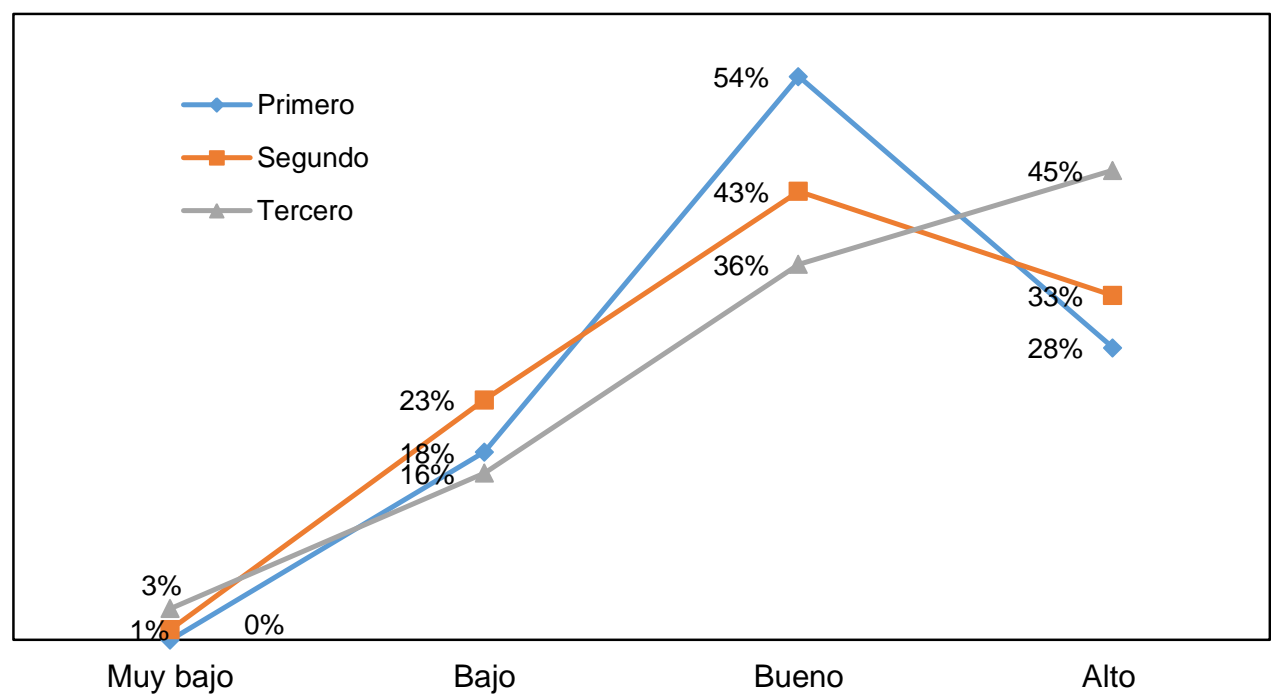

Fig. 1: Comparativo del factor de la supervisión académica de los tutores y el año escolar

Tabla 2: Prueba de contraste de Omnibus - factor 1

\begin{tabular}{|c|c|c|}
\hline $\begin{array}{c}\text { Chi-cuadrado } \\
\text { de la razón de } \\
\text { verosimilitudes }\end{array}$ & gl & sig \\
\hline 22.197 & 7 & 0.002 \\
\hline
\end{tabular}

Tabla 3: Contraste de los efectos del modelo - factor 1

\begin{tabular}{|c|c|c|c|}
\hline \multirow{2}{*}{ Origen } & \multicolumn{3}{|c|}{ Tipo III } \\
\cline { 2 - 4 } & Chi-cuadrado de wald & gl & Sig. \\
\hline (Intersección) & 737.723 & 1 & 0.000 \\
\hline Grado & 5.437 & 2 & 0.066 \\
\hline Genero & 1.157 & 1 & 0.282 \\
\hline Tutor & 14.78 & 4 & 0.005 \\
\hline
\end{tabular}

\section{Factor 2: Autoestima y Motivación}

En la investigación se les preguntó a los adolescentes si sus tutores mostraban manifestaciones de cariño hacia ellos y de forma frecuente les decían que los querían y que los apoyaban, y $84 \%$ de los adolescentes indicó que si y el $6 \%$ dijo no sentir esas muestras de afecto. Por otra parte, el $92 \%$ de los encuestados enfatizó que sus tutores los motivan para salir adelante, mientras que el $2 \%$ no perciben ese tipo de estímulos emocionales y el $6 \%$ no demostró ninguna tendencia. Además, el 5\% indicaron no estar de acuerdo en que su tutor los motive para que lleguen temprano a la escuela, únicamente el $83 \%$ señaló estar de acuerdo, el $12 \%$ indicó no estar de acuerdo ni en desacuerdo. Por otro lado, el $87 \%$ de los niños está de acuerdo en que sus tutores se preocupan por sus avances académicos en la escuela, sólo el $3 \%$ de ellos no está de acuerdo y el resto no muestra tendencia.

También se preguntó si los alumnos sentían que sus padres procuraban que contarán con el material necesario para las actividades que se llevan a cabo en la escuela, el $8 \%$ dijo que no, mientras que el $80 \%$ enfatizó que sí y el $12 \%$ no mostró preferencia. Asimismo, el $80 \%$ de los adolescentes dijo que cuando llegan a casa después de salir de la escuela hay alguien esperándolos, el $80 \%$ señaló que sí y el $8 \%$ indicó que no. Se llevó a cabo el modelo lineal generalizado en donde se encontró que la prueba de contraste de Omnibus obtuvo una $p=0.061>0.05$ que indica que no hay diferencias lo que significa que los coeficientes de las variables no son estadísticamente significativos (ver tabla 4). Sin embargo, los resultados de la tabla de los contrastes del modelo indican que la variable del tutor tiene efectos significativos sobre el modelo $(p=$ $0.043<0.05$ ), revisando los resultados de las medias marginales arrojan que las madres al parecer son las que contribuyen en incrementar la autoestima y la motivación de los jóvenes $(\bar{x}=30.67)$, en segundo lugar los papás $(\bar{x}=30.67)$ y en tercer lugar los abuelitos $(\bar{x}=30.44)$ (ver tabla 5$)$. 
Se calculó una escala con los siete ítems, se tomó la decisión de dejar en tres categorías la escala, "bajo", "bueno" y "alto", se aplicó una tabla de contingencia para analizar el cruce de las variables, se encontró que en los tres años escolares los adolescentes perciben que sus tutores contribuyen en incrementar la autoestima y motivación.

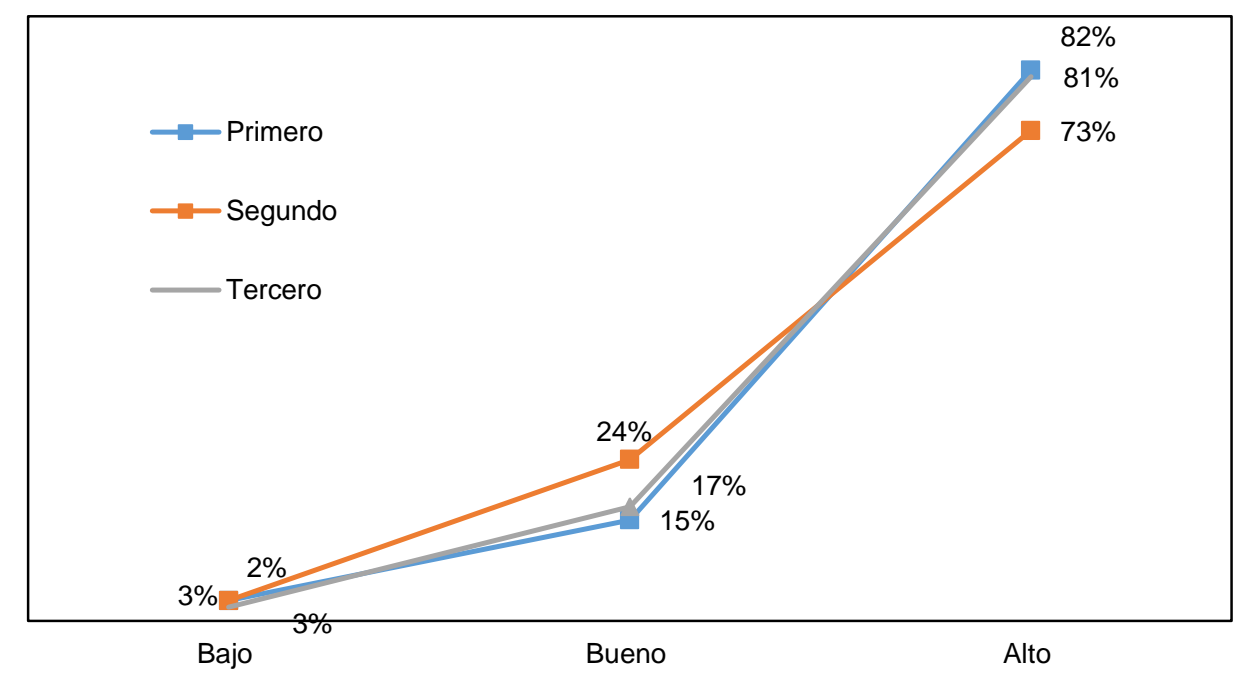

Fig. 2: Comparativo del factor de autoestima - motivación y el año escolar

Tabla 4: Prueba de contraste de Omnibus - factor 2

\begin{tabular}{|c|c|c|}
\hline $\begin{array}{c}\text { Chi-cuadrado } \\
\text { de la razón de } \\
\text { verosimilitudes }\end{array}$ & gl & sig \\
\hline 13.483 & 7 & 0.061 \\
\hline
\end{tabular}

Tabla 5: Contraste de los efectos del modelo - factor 2

\begin{tabular}{|c|c|c|c|}
\hline \multirow{2}{*}{ Origen } & \multicolumn{3}{|c|}{ Tipo III } \\
\cline { 2 - 4 } & Chi-cuadrado de wald & gl & Sig. \\
\hline (Intersección) & 2370.058 & 1 & 0.000 \\
\hline Grado & 3.553 & 2 & 0.169 \\
\hline Genero & 0.221 & 1 & 0.639 \\
\hline Tutor & 9.858 & 4 & 0.043 \\
\hline
\end{tabular}

\section{Factor 3: Castigos y Atención a los Citatorios Importantes}

En esta dimensión únicamente se agruparon dos variables, los castigos y el apoyo de parte del tutor para la realización de las tareas. Se encontró que el $63 \%$ de los adolescentes afirmaron recibir algún tipo de sanción de parte de sus tutores en caso de obtener malas calificaciones, por su parte el $20 \%$ señaló no recibir castigos y el resto no mostro ninguna tendencia. Por último, el $85 \%$ está de acuerdo en que sus padres atienden los citatorios y acuden a las juntas importantes, sólo un $5 \%$ estuvo en desacuerdo. Se hizo un comparativo de los años escolares y el factor, se observó que los estudiantes de primer semestre califican mejor el factor y se detectó una disminución con los jóvenes de tercer año.

Se llevó a cabo el modelo lineal generalizado con el factor tres y las variables: a) grado, b) género y c) tutor, la prueba de contraste de Omnibus obtuvo como resultado una $p=0.001<0.05$ que indica que por lo menos uno de los coeficientes calculados para el modelo es estadísticamente significativo (ver tabla 6), estudiando los efectos del contraste del modelo (ver tabla 7), se encontró que las variables del género $(p=0.000<0.05)$ y género $(p=0.05<=0.05)$ tienen efecto significativo sobre el modelo, observando los resultados de las medias marginales se tienen que en el caso del grado escolar, en el primer año el puntaje de la media marginal es mayor $(\bar{x}=8.52)$, en el tercer año $(\bar{x}=7.86)$ y en el segundo año $(\bar{x}=7.79)$. Por otra parte, se halló que el género también obtuvo diferencias, las mujeres obtuvieron mayor puntuación $(\bar{x}=8.21)$ con respecto a los hombres $(\bar{x}=7.90)$ 


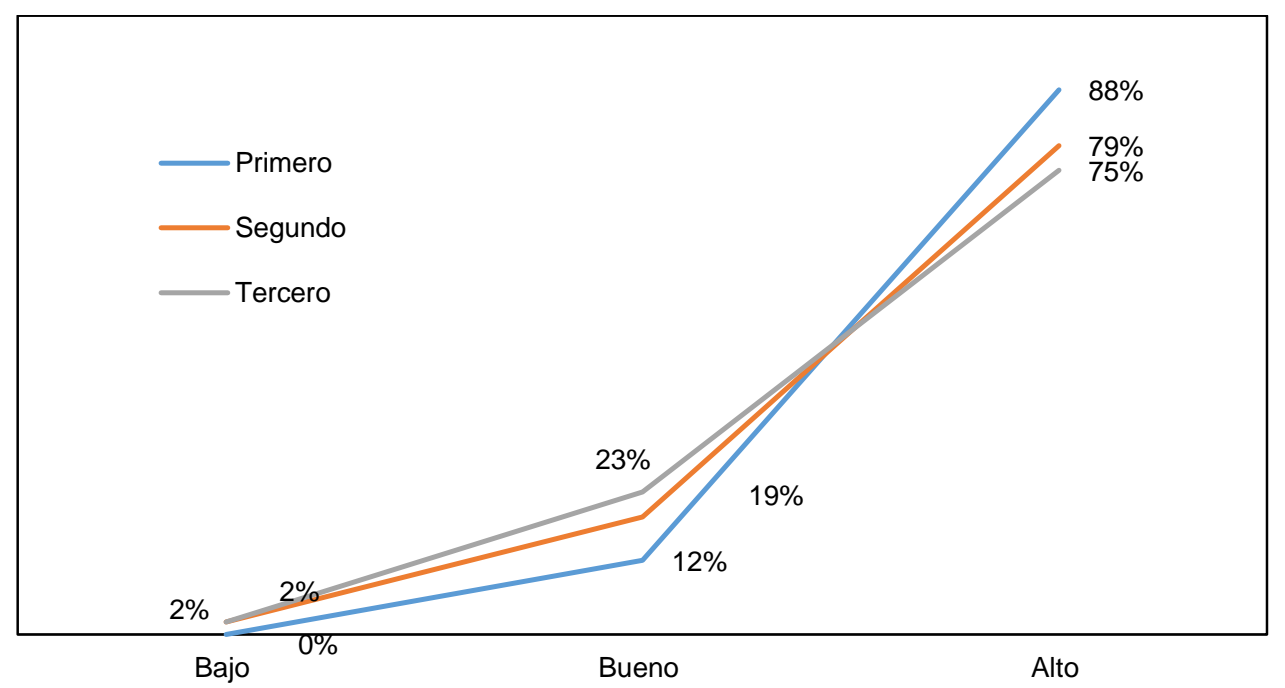

Fig. 3: Comparativo del factor de castigos y atención a los citatorios importantes y el año escolar

Tabla 6: Prueba de contraste de Omnibus - factor 3

\begin{tabular}{|c|c|c|}
\hline $\begin{array}{c}\text { Chi-cuadrado } \\
\text { de la razón de } \\
\text { verosimilitudes }\end{array}$ & gl & sig \\
\hline 25.009 & 7 & 0.001 \\
\hline
\end{tabular}

Tabla 7: Contraste de los efectos del modelo - factor 3

\begin{tabular}{|c|c|c|c|}
\hline \multirow{2}{*}{ Origen } & \multicolumn{3}{|c|}{ Tipo III } \\
\cline { 2 - 4 } & Chi-cuadrado de wald & gl & Sig. \\
\hline (Intersección) & 1303.459 & 1 & 0.000 \\
\hline Grado & 18.367 & 2 & 0.000 \\
\hline Genero & 3.809 & 1 & 0.051 \\
\hline Tutor & 2.708 & 4 & 0.608 \\
\hline
\end{tabular}

\section{DISCUSIÓN FINAL}

El éxito académico de los adolescentes es un fenómeno que involucra diversos elementos que interactúan buscando el bienestar y el mejor desarrollo de los jóvenes. En la investigación se encontró que la madre sigue siendo considerada como principal tutor en la mayoría de los jóvenes que participaron en el estudio, lo anterior refuerza lo señalado por Schlechter y Milevsky (2010) que mencionan que los papás mantienen una mínima participación en la educación de los hijos, por lo que se recomienda determinar formas de alentar a que los padres asistan a las reuniones de los profesores. Sin embargo, lo anterior es algo que se puede confrontar, ya que en este trabajo a pesar de que las madres son percibidas como los tutores titulares, los papás son lo que llevan a cabo la mayor supervisión académica con respecto a la madre, lo que significa que es a pesar de la responsabilidad que tienen las mamás, el papá tiene autoridad para llevar a cabo ese tipo de evaluaciones y verificación a sus hijos.

Por otra parte, el elemento de la autoestima y motivación es fundamental para el progreso de los estudiantes, los resultados que se derivan de este factor indican que los jóvenes de la escuela secundaria que se analizó son chicos que sienten el cariño y el apoyo de sus padres, aunado a la percepción que ellos tienen con relación a la motivación, siendo ésta un aliciente para salir adelante. Al respecto, Stanard, et. al. (2010), enfatizan que las familias cuyos lazos emocionales son fuertes influyen de forma positiva en los adolescentes, además coadyuvan a otorgarle un valor importante a la educación, por lo que se puede motivar para incrementar un interés más alto en la escuela y el esfuerzo académico, asimismo este tipo de familias refuerza la autoestima de tal manera que los jóvenes tienen la capacidad de recibir malas críticas de sus compañeros y saben cómo evitarlas. 
Por lo que estamos de acuerdo con las sugerencias hechas por los investigadores (Coronado, et. al., 2011) que enfatizan que es necesario incentivar que la familia participe de forma activa ya que es considerado como órgano solidario que apoya el desarrollo del educando. Lo anterior, es soportado por Stanard et al. (2010), que indican que la familia representa un rol importante en crecimiento del adolescente, en ausencia de los lazos familiares el estudiante es susceptible de las influencias negativas externas. También, la motivación académica se incrementa cuando los jóvenes perciben que sus padres apoyan y responden sus necesidades académicas aunado a que la comprensión de los procesos familiares predicen el rendimiento académico (Henry, et. al., 2011; Mulvaney y Morrissey, 2012). Por último, se reconoce que las variables personales de los alumnos y la protección que brinda la resiliencia de la familia permite incrementar la esperanza, la autoestima y el logro académico de los adolescentes (Çelik, et.al., 2014).

\section{CONCLUSIONES}

Las implicaciones para la escuela secundaria que fue objeto de estudio son diversas, en principio es reconocer que los jóvenes que asisten a ella están abrigados por sus madres quienes representan en mayor medida la figura del tutor, además de que son ellas las que realizan un extraordinario trabajo que apoya en fortalecer su motivación y autoestima, sin embargo se requiere afianzar estrategias relacionadas con el involucramiento de ellas en el tema de la supervisión académica, pero sin olvidarse el compromiso de los papás. Por otro lado, es imprescindible que los padres trabajen con intervenciones adecuadas para fomentar el interés en la escuela de parte de los adolescentes, ya que esto incrementa la confianza, disminuye la incertidumbre y afianza el sentido de la importancia que tiene la educación para forjar su futuro.

Además, se recomienda que la escuela trabaje con los padres de familia en diversos temas relacionados con las esferas de la vida de los adolescentes para que de esta forma incrementen la confianza y compromiso con su trayactoria académica. Lo anterior refuerza lo que pretende el Programa Sectorial de Educación 2013-2018 que reconoce que para alcanzar la calidad de la educación es necesario que los actores colaboren en conjunto para que la comunicación sea fluida y de esta forma se consiga entender la tarea educativa, a su vez detectar problemas de rendimiento académico y aplicar estrategias orientadas en apoyar a los jóvenes que presentan deficiencias en algunas áreas del conocimiento.

Por último, los profesores son un elemento clave en la formación académica de un adolescente, por lo tanto, es necesario que exista una interacción constante entre tutores y maestros, aunando a la necesidad de que ambos reciban capacitación en temas que trastocan los problemas actuales a los que se enfrentan los jóvenes en México. Las límitaciones del estudio se detectaron en las variables independientes del tipo sociodemográficas, las cuales se sugieren estudiar.

\section{AGRADECIMIENTOS}

Los autores agradecen el apoyo al Instituto Politénico Nacional y a la Secretaría de Investigación y Posgrado, producto obtenido a partir del proyecto de Investigación con número de Registro SIP 20164799.

\section{REFERENCIAS}

Areepattamannil, S.; Relationship between academic motivation and mathematics achievement among Indian adolescents in Canada and India. The Journal of general psychology, 141(3), 247-262 (2014)

Arostegui, I., N. Beloki y L. Darretxe; La participación de las familias y de otros miembros de la comunidad como estrategia de éxito en las escuelas. RIEE. Revista Iberoamericana de Evaluación Educativa, 6(2), 187$200(2013)$

Booth, M.Z. y J.M. Gerard; Self-esteem and academic achievement: a comparative study of adolescent students in England and the United States. Compare: A Journal of Comparative and International Education, 41(5), 629-648 (2011)

Braun, O., O. Idan, B. Lindström y M. Margalit; Salutogenesis Sense of Coherence in Adolescence. In The Handbook of Salutogenesis. Springer International Publishing, 123-136 (2017)

Carvallo, M., J. Caso Niebla y L. Á. Contreras; Estimación del efecto de variables contextuales en el logro académico de estudiantes de Baja California. Revista electrónica de investigación educativa, 9(2), 1-15 (2007) 
Çelik, D. A., F. Çetin y E. Tutkun; The role of proximal and distal resilience factors and locus of control in understanding hope, self-esteem and academic achievement among Turkish pre-adolescents. Current Psychology, 34(2), 321-345 (2015)

Cerezo, M.T., P.F. Casanova, J. Manuel y M. De la Villa Carpio; Estilos educativos paternos y estrategias de aprendizaje en alumnos de Educación Secundaria. European Journal of Education and Psychology, 4(1) (2015)

Chaparro, C.L., C. González y J. Caso; Familia y rendimiento académico: configuración de perfiles estudiantiles en secundaria. Revista electrónica de investigación educativa, 18(1), 53-68 (2016)

Cho, S. y J.R. Campbell; Differential influences of family processes for scientifically talented individuals' academic achievement along developmental stages. Roeper Review, 33(1), 33-45 (2010)

Coronado, M., C.C. Torres y A.M. Montilla; Dos Pilares Indispensables Para Afrontar El Fracaso Escolar Actual: Familias y Centros Educativos. International Journal of Development and Educational Psychology 5(1), 623-32 (2011)

Crede, J., N. Wirthwein, N. McElvany y R. Steinmayr; Adolescents' academic achievement and life satisfaction: the role of parents' education. Frontiers in psychology, 6, 52 (2015)

García, A.O., L.F. Soto y R.G. Ochoa; Papá y mamá: dos maneras de ver la vida... y una misma educación. Enseñanza e Investigación en Psicología, 18(1), 51-67 (2013)

Gutman, L. M. y V.C. McLoyd; Parents' management of their children's education within the home, at school, and in the community: An examination of African-American families living in poverty. The Urban Review, 32(1), 1-24 (2000)

Henry, C.S., S.W. Plunkett y T. Sands; Family structure, parental involvement, and academic motivation in Latino adolescents. Journal of Divorce \& Remarriage, 52(6), 370-390 (2011)

Ibarra, E., J. García y H. Manuel; La evolución del autoconcepto académico en adolescentes. Revista mexicana de investigación educativa, 21(68), 45-70 (2016)

Kilian, B., M. Hofer y C. Kuhnle; Conflicts between on-task and off-task behaviors in the classroom: the influences of parental monitoring, peer value orientations, students' goals, and their value orientations. Social psychology of education, 16(1), 77-94 (2013)

Kim, S.Y., Y. Wang, Q. Chen, Y. Shen y Y. Hou; Parent-Child Acculturation Profiles as Predictors of Chinese American Adolescents' Academic Trajectories. Journal of youth and adolescence, 44(6), 1263-1274 (2014)

Kristjansson, A. L. y I.D. Sigfusdottir; The role of parental support, parental monitoring, and time spent with parents in adolescent academic achievement in lceland: A structural model of gender differences. Scandinavian journal of educational research, 53(5), 481-496 (2009)

Latunde, Y.; Introduction: Setting the Stage for Success. Chapter Research in Parental Involvement, en la web: https://link.springer.com/chapter/10.1057/978-1-137-59146-3_1, pp. 3-20, Date: 26 October (2016)

Lazaridou, A. y A. Gravani Kassida; Involving parents in secondary schools: principals' perspectives in Greece. International Journal of Educational Management, 29(1), 98-114 (2015)

Loose, F.; Individualism: Valued differently by parents and teachers of primary, junior high and high school students. Social Psychology of Education, 11(2), 117-131 (2008)

López, O., C. Hederich y Á. Camargo; Cognitive Style and Academic Achievement. Educación y Educadores, 14(1), 67-82 (2011)

Mulvaney, M.K. y R.A. Morrissey; Parenting beliefs and academic achievement across African-American and Caucasian family contexts. Early Child Development and Care, 182(9), 1105-1124 (2012)

Rao, M. B. y M. Mehta; Academic Choices and Perceptions of Success by Students in Mumbai: An Analysis of Socio-Cultural Factors. In Success in Higher Education (pp. 301-314). Springer Singapore (2017)

Räty, H.; School's out: A comprehensive follow-up study on parents' perceptions of their child's school. European Journal of Psychology of Education, 25(4), 493-506 (2010) 
Ricard, N. C. y L.G. Pelletier; Dropping out of high school: The role of parent and teacher self-determination support, reciprocal friendships and academic motivation. Contemporary Educational Psychology, 44, 32-40 (2016)

Schlechter, M. y A. Milevsky; Parental level of education: associations with psychological well-being, academic achievement and reasons for pursuing higher education in adolescence. Educational Psychology, 30(1), 1-10 (2010)

Simon, B.; High school outreach and family involvement. Social Psychology of Education, 7(2), 185-209 (2004)

Stanard, P., F.Z. Belgrave, M.A. Corneille, K.D. Wilson y K. Owens; Promoting academic achievement: The role of peers and family in the academic engagement of African American adolescents. Journal of prevention \& intervention in the community, 38(3), 198-212 (2010)

Toro, J. W., L.F. Guzman y J. Jaramillo; Concepciones acerca de la motivación en el aprendizaje y logro académico en niños y niñas de grado quinto de primaria de la Institución Educativa San Fernando Cuba. Textos y Sentidos 7(1), 56-87 (2013)

Wilder, S.; Effects of parental involvement on academic achievement: a meta-synthesis. Educational Review, 66(3), 377-397 (2014) 